NASA/TM-2002-211583

ICOMP-2002-04
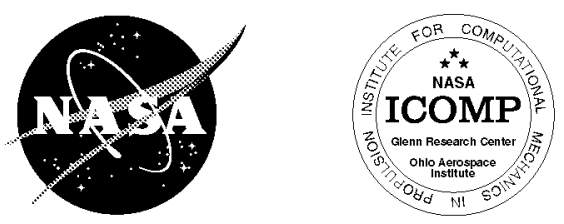

AIAA-2002-2438

\title{
Towards Arbitrary Accuracy Inviscid Surface Boundary Conditions
}

Rodger W. Dyson

Glenn Research Center, Cleveland, Ohio

Ray Hixon

Institute for Computational Mechanics in Propulsion, Cleveland, Ohio and

University of Toledo, Toledo, Ohio

July 2002 
The NASA STI Program Office ... in Profile

Since its founding, NASA has been dedicated to the advancement of aeronautics and space science. The NASA Scientific and Technical Information (STI) Program Office plays a key part in helping NASA maintain this important role.

The NASA STI Program Office is operated by Langley Research Center, the Lead Center for NASA's scientific and technical information. The NASA STI Program Office provides access to the NASA STI Database, the largest collection of aeronautical and space science STI in the world. The Program Office is also NASA's institutional mechanism for disseminating the results of its research and development activities. These results are published by NASA in the NASA STI Report Series, which includes the following report types:

- TECHNICAL PUBLICATION. Reports of completed research or a major significant phase of research that present the results of NASA programs and include extensive data or theoretical analysis. Includes compilations of significant scientific and technical data and information deemed to be of continuing reference value. NASA's counterpart of peerreviewed formal professional papers but has less stringent limitations on manuscript length and extent of graphic presentations.

- TECHNICAL MEMORANDUM. Scientific and technical findings that are preliminary or of specialized interest, e.g., quick release reports, working papers, and bibliographies that contain minimal annotation. Does not contain extensive analysis.

- CONTRACTOR REPORT. Scientific and technical findings by NASA-sponsored contractors and grantees.
- CONFERENCE PUBLICATION. Collected papers from scientific and technical conferences, symposia, seminars, or other meetings sponsored or cosponsored by NASA.

- SPECIAL PUBLICATION. Scientific, technical, or historical information from NASA programs, projects, and missions, often concerned with subjects having substantial public interest.

- TECHNICAL TRANSLATION. Englishlanguage translations of foreign scientific and technical material pertinent to NASA's mission.

Specialized services that complement the STI Program Office's diverse offerings include creating custom thesauri, building customized data bases, organizing and publishing research results ... even providing videos.

For more information about the NASA STI Program Office, see the following:

- Access the NASA STI Program Home Page at http://www.sti.nasa.gov

- E-mail your question via the Internet to help@sti.nasa.gov

- Fax your question to the NASA Access Help Desk at 301-621-0134

- Telephone the NASA Access Help Desk at 301-621-0390

- Write to: NASA Access Help Desk NASA Center for AeroSpace Information 7121 Standard Drive Hanover, MD 21076 
NASA/TM-2002-211583

ICOMP-2002-04
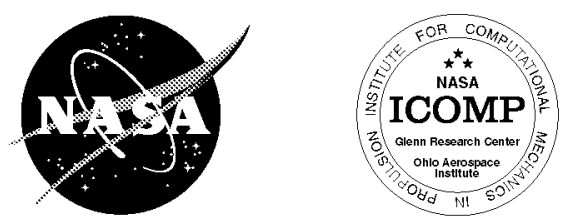

AIAA-2002-2438

\title{
Towards Arbitrary Accuracy Inviscid Surface Boundary Conditions
}

\author{
Rodger W. Dyson \\ Glenn Research Center, Cleveland, Ohio \\ Ray Hixon \\ Institute for Computational Mechanics in Propulsion, Cleveland, Ohio \\ and \\ University of Toledo, Toledo, Ohio
}

Prepared for the

Eighth Aeroacoustics Conference

cosponsored by the American Institute of Aeronautics and Astronautics and the Confederation of European Aerospace Societies

Breckenridge, Colorado, June 17-19, 2002

National Aeronautics and

Space Administration

Glenn Research Center

July 2002 
This report is a formal draft or working paper, intended to solicit comments and ideas from a technical peer group.

This report contains preliminary findings, subject to revision as analysis proceeds.

This report is a preprint of a paper intended for presentation at a conference. Because of changes that may be made before formal publication, this preprint is made available with the understanding that it will not be cited or reproduced without the permission of the author.

Available from

NASA Center for Aerospace Information 7121 Standard Drive

Hanover, MD 21076
National Technical Information Service 5285 Port Royal Road Springfield, VA 22100

Available electronically at htp://gltrs.grc.nasa.gov/GLTRS 


\title{
Towards Arbitrary Accuracy Inviscid Surface Boundary Conditions
}

\author{
Rodger W. Dyson* \\ NASA Glenn Research Center at Lewis Field, Cleveland, OH 44135 \\ Ray Hixon ${ }^{\dagger}$ \\ Institute for Computational Mechanics in Propulsion, University of Toledo, Toledo, OH 43606
}

\begin{abstract}
Inviscid nonlinear surface boundary conditions are currently limited to $3^{\text {rd }}$ order accuracy in time for non-moving surfaces and actually reduce to $1^{\text {st }}$ order in time when the surfaces move. For steady-state calculations it may be possible to achieve higher accuracy in space, but high accuracy in time is required for efficient simulation of multiscale unsteady phenomena. A surprisingly simple technique is shown here that can be used to correct the normal pressure derivatives of the flow at a surface on a Cartesian grid so that arbitrarily high order time accuracy is achieved in idealized cases. This work demonstrates that nonlinear high order time accuracy at a solid surface is possible and desirable, but it also shows that the current practice of only correcting the pressure is inadequate.
\end{abstract}

\section{Introduction}

$\mathbf{H}$ IGH order accuracy is required for efficient aeroacoustic simulations. ${ }^{1-3}$ Recently, it has been demonstrated ${ }^{4,5}$ that there is an optimal range of design accuracies in which the best efficiency and solution accuracy is obtained. The optimal value depends upon the length of the simulation and the level of accuracy required. For example, nonlinear inviscid aeroacoutics simulations with $6^{\text {th }}$ order accuracy in space and time is optimal for propagating a wave five wavelengths with absolute error less than $10^{-6}$. Unfortunately, this is not typically achieved in practice because of the solid boundary conditions ${ }^{6}$ which may have high order spatial accuracy, but rarely exceed $2^{\text {nd }}$ order time accuracy. This severely limits the efficiency of time marching methods.

This paper demonstrates why most surface boundary approaches are typically only $1^{\text {st }}$ order accurate in time and it presents a surprisingly simple procedure for correcting the normal pressure derivatives to achieve any order of time accuracy in special cases. The basic idea follows the work of $\mathrm{Tam}^{7,8}$ in which walls are modeled by pressure corrections; and follows the work of Hixon ${ }^{9}$ in which the pressure corrections are treated separately from flow variables to greatly simplify their nonlinear implementation; and finally, those contributions are combined with Goodrich's boundary treatment ${ }^{10}$ to produce arbitrary time accuracy at a

\footnotetext{
*Aerospace Engineer, Acoustics Branch, Structures and Acoustics Division, Research and Technology Directorate.

†Senior Research Associate, Member AIAA

Copyright (C) 2002 by the American Institute of Aeronautics and Astronautics, Inc. No copyright is asserted in the United States under Title 17, U.S. Code. The U.S. Government has a royaltyfree license to exercise all rights under the copyright claimed herein for Governmental Purposes. All other rights are reserved by the copyright owner.
}

surface.

The usefulness of these ideas are currently limited to cases in which the pressure corrections are much larger than the density and velocity corrections. Nonetheless, the procedures shown here demonstrate the feasibility and efficiency of increasing the time accuracy at surfaces.

\section{Problem Description}

The following form of the two-dimensional nonlinear Euler equations are used:

$$
\begin{aligned}
\frac{\partial \rho}{\partial t}+u \frac{\partial \rho}{\partial x}+v \frac{\partial \rho}{\partial y}+\rho\left(\frac{\partial u}{\partial x}+\frac{\partial v}{\partial y}\right) & =Q_{1} \\
\frac{\partial p}{\partial t}+u \frac{\partial p}{\partial x}+v \frac{\partial p}{\partial y}+\gamma p\left(\frac{\partial u}{\partial x}+\frac{\partial v}{\partial y}\right) & =Q_{2} \\
\frac{\partial(\rho u)}{\partial t}+\frac{\partial\left(\rho u^{2}+p\right)}{\partial x}+\frac{\partial(\rho u v)}{\partial y} & =Q_{3} \\
\frac{\partial(\rho v)}{\partial t}+\frac{\partial(\rho u v)}{\partial x}+\frac{\partial\left(\rho v^{2}+p\right)}{\partial y} & =Q_{4}
\end{aligned}
$$

At the solid boundary we also have the following infinite set of boundary conditions: ${ }^{10}$

$$
\frac{\partial^{\alpha}(\vec{V} \cdot \hat{n})}{\partial t^{\alpha}}=\frac{\partial^{\alpha} Q_{5}}{\partial t^{\alpha}} \forall \alpha:(\alpha \geq 0)
$$

Ordinarily, the source terms $\left(Q_{1}, Q_{2}, Q_{3}, Q_{4}, Q_{5}\right)$ are zero. But, for testing purposes, these terms will be modified so that the following analytical solution is defined: ${ }^{5}$

$$
\begin{aligned}
& \rho(x, y, t)=a_{1} \cos \left(k_{x} \pi x\right) \cos \left(k_{y} \pi y\right) \cos \left(k_{t} \pi t\right)+c_{1} \\
& u(x, y, t)=a_{2} \cos \left(k_{x} \pi x\right) \cos \left(k_{y} \pi y\right) \cos \left(k_{t} \pi t\right)+c_{2} \\
& v(x, y, t)=a_{3} \cos \left(k_{x} \pi x\right) \cos \left(k_{y} \pi y\right) \cos \left(k_{t} \pi t\right)+c_{3} \\
& p(x, y, t)=a_{4} \cos \left(k_{x} \pi x\right) \cos \left(k_{y} \pi y\right) \cos \left(k_{t} \pi t\right)+c_{4}
\end{aligned}
$$


Due to the large number of derivatives used in what follows, the following shorthand notation will be used:

$$
\stackrel{\chi}{C}_{a, b, k}^{f}=\frac{\partial^{a+b+k} f(x, y, t)}{\partial x^{a} y^{b} t^{k}}
$$

where $\chi$, when used, will indicate: updated $(U)$, new $(N)$, old $(O)$, or correction $(C)$.

\section{Maintaining High Accuracy at Boundary}

This work uses high accuracy Hermitian Modified Expansion Solution Approximation (MESA) methods because high time accuracy at a surface requires correcting the spatial cross derivatives of the flow variables, many of which the MESA scheme explicitly includes on the grid. Any method could be used as long as the effect of cross derivative corrections are consistently applied. In Fig. 1, the absolute error of the numerical wavenumber for the derivative of a harmonic function, $f(x)=\exp ^{i k x}$, is plotted by points per wavelength (PPW) as done in Ref. ${ }^{11}$ The extraordinary resolution of the two-point MESA schemes allows one to use fewer grid points and larger time steps, resulting in significant computational efficiency for problems requiring high physical accuracy as shown in Fig. 1. 5,15 However, maintaining high accuracy at surfaces, ${ }^{12}$ particularly when waves are being scattered, ${ }^{13}$ is required to maintain this high efficiency.

Unfortunately, most inviscid surface boundary conditions are at most $2^{\text {nd }}$ order accurate in time since only the following conditions:

$$
\frac{\partial^{\alpha}(\vec{V} \cdot \hat{n})}{\partial t^{\alpha}}=0 \forall \alpha:(\alpha=0,1)
$$

are used when in fact Eq. (5) is true for any $\alpha$.

As an example, the numerical time advancement of the pressure on a surface expressed as a Taylor series is:

$$
\begin{aligned}
& p(x, y, t+\Delta t)= \\
& \quad C_{0,0,0}^{p}+C_{0,0,1}^{p} \Delta t+C_{0,0,2}^{p} \frac{\Delta t^{2}}{2 !}+\ldots
\end{aligned}
$$

And from the governing equations Eqs. (1, 2, 3, 4) we can convert the series' time derivatives into space derivatives. For example:

$$
\begin{aligned}
& -C_{0,0,1}^{p}= \\
& C_{0,0,0}^{u} C_{1,0,0}^{p}+C_{0,0,0}^{v} C_{0,1,0}^{p}+\gamma C_{0,0,0}^{p}\left(C_{1,0,0}^{u}+C_{0,1,0}^{v}\right)
\end{aligned}
$$

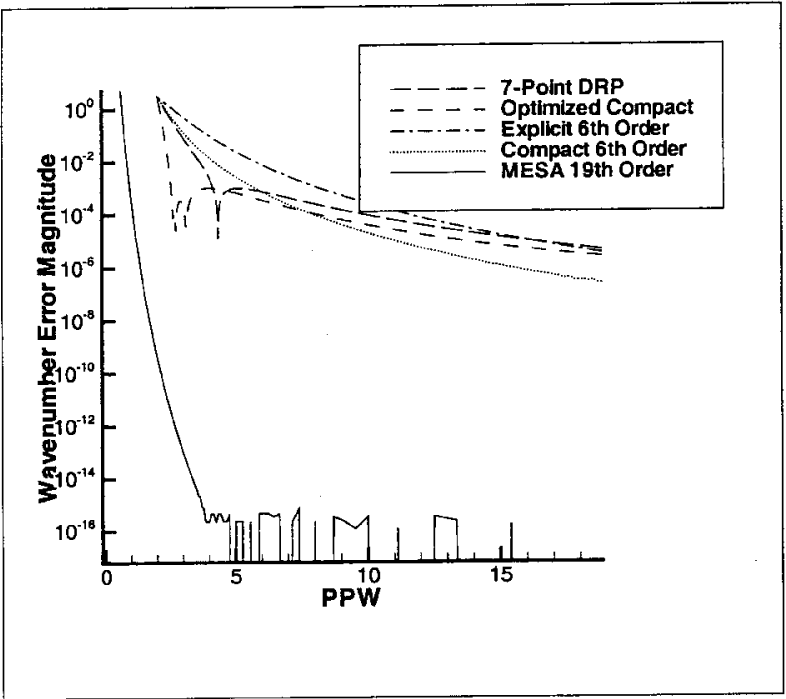

a) MESA scheme compares well to popular techniques

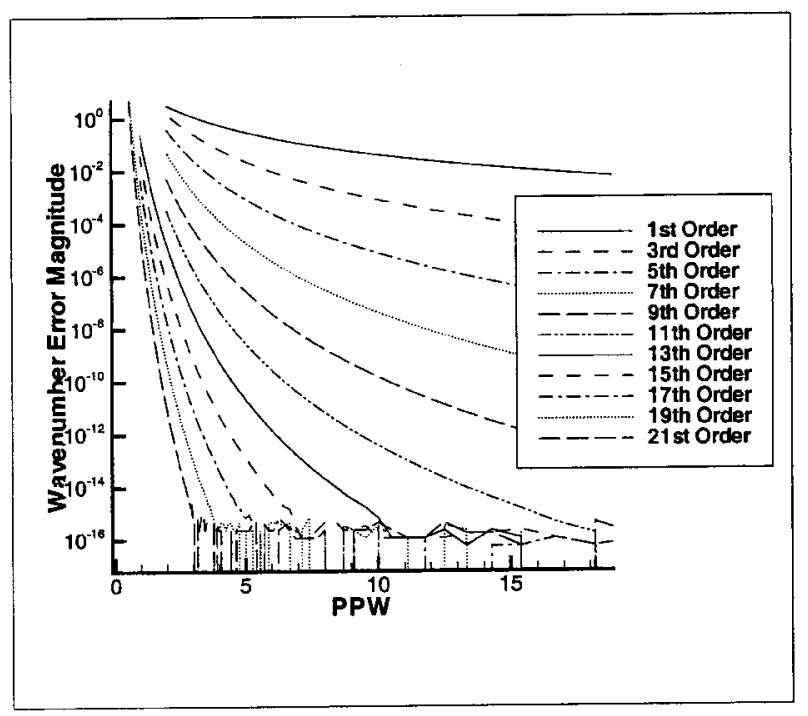

b) Resolution of Two-Point MESA schemes

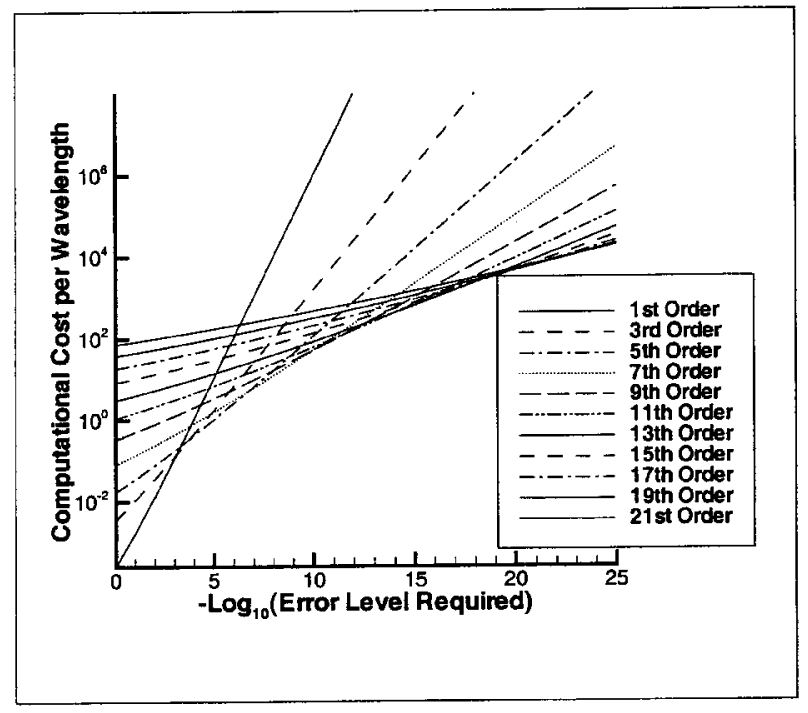

c) Efficiency of Nonlinear Euler Solver

Fig. 1 
and

$$
\begin{aligned}
& -C_{0,0,2}^{p}= \\
& C_{1,0,0}^{p}\left(C_{0,0,0}^{u} C_{1,0,0}^{u}+C_{0,0,0}^{v} C_{0,1,0}^{u}+C_{0,0,0}^{1 / p} C_{1,0,0}^{p}\right)+ \\
& C_{0,0,0}^{u}\left(C_{1,0,0}^{u} C_{1,0,0}^{p}+C_{0,0,0}^{u} C_{2,0,0}^{p}+C_{1,0,0}^{v} C_{0,1,0}^{p}+\right. \\
& C_{0,0,0}^{v} C_{1,1,0}^{p}+\gamma C_{1,0,0}^{p}\left(C_{1,0,0}^{u}+C_{0,1,0}^{v}\right)+ \\
& \left.\gamma C_{0,0,0}^{p}\left(C_{2,0,0}^{u}+C_{1,1,0}^{v}\right)\right)+ \\
& C_{0,1,0}^{p}\left(C_{0,0,0}^{u} C_{1,0,0}^{v}+C_{0,0,0}^{v} C_{0,1,0}^{v}+C_{0,0,0}^{1 / \rho} C_{0,1,0}^{p}\right)+ \\
& C_{0,0,0}^{v}\left(C_{0,1,0}^{u} C_{1,0,0}^{p}+C_{0,0,0}^{u} C_{1,1,0}^{p}+C_{0,1,0}^{v} C_{0,1,0}^{p}+\right. \\
& C_{0,0,0}^{v} C_{0,2,0}^{p}+\gamma C_{0,1,0}^{p}\left(C_{1,0,0}^{u}+C_{0,1,0}^{v}\right)+ \\
& \left.\gamma C_{0,0,0}^{p}\left(C_{1,1,0}^{u}+C_{0,2,0}^{v}\right)\right)+ \\
& \gamma\left(C_{1,0,0}^{u}+C_{0,1,0}^{v}\right)\left(C_{0,0,0}^{u} C_{1,0,0}^{p}+C_{0,0,0}^{v} C_{0,1,0}^{p}+\right. \\
& \left.\gamma C_{0,0,0}^{p}\left(C_{0,0,0}^{v} C_{0,1,0}^{p}\right)\right)+ \\
& \gamma C_{0,0,0}^{p}\left(-\left(C_{1,0,0}^{u} C_{1,0,0}^{u}+C_{0,0,0}^{u} C_{2,0,0}^{u}+C_{1,0,0}^{v} C_{0,1,0}^{u}+\right.\right. \\
& \left.\left.C_{0,0,0}^{v} C_{1,1,0}^{u}+C_{1,0,0}^{u} / \rho C_{1,0,0}^{p}+C_{0,0,0}^{u} C_{2,0,0}^{p}+\right)^{p}\right)+ \\
& \gamma C_{0,0,0}^{p}\left(-\left(C_{0,1,0}^{u} C_{1,0,0}^{v}+C_{0,0,0}^{u} C_{1,1,0}^{v}+C_{0,1,0}^{v} C_{0,1,0}^{v}+\right.\right. \\
& C_{0,0,0}^{v} C_{0,2,0}^{v}+C_{0,1,0}^{v}+\rho \\
& \quad
\end{aligned}
$$

These equations clearly show that for $2^{\text {nd }}$ order time accuracy we must have the correct first order spatial derivatives of the pressure and velocities at the surface. In particular, Eq. (5) with $\alpha=1$ provides a constraint on these $1^{s t}$ order spatial derivatives by converting the time derivatives to space derivatives again using Eqs. (31, 31):

$$
\begin{aligned}
& -\eta_{x}\left(\begin{array}{l}
C_{0,0,0}^{u} C_{1,0,0}^{u}+C_{0,0,0}^{v} C_{0,1,0}^{u}+C_{0,0,0}^{1 / \rho} C_{1,0,0}^{p} \\
-\eta_{y}
\end{array}\left(\begin{array}{l}
C_{0,0,0}^{u} C_{1,0,0}^{v}+C_{0,0,0}^{v} C_{0,1,0}^{v}+C_{0,0,0}^{1 / \rho} C_{0,1,0}^{p}
\end{array}\right)=0\right.
\end{aligned}
$$

which if not true, implies the time accuracy is only $1^{\text {st }}$ order.

Similarly, the boundary condition Eq. (5) with $\alpha=$ 2 will provide a constraint on the first and second order spatial derivatives (including density), which if not true, will reduce the numerical method to below $3^{\text {rd }}$ order time accuracy. And in general, one must enforce Eq. (5) for all $\alpha$ up to the desired time accuracy. Note, however, in the special case of a nonmoving inviscid wall that the even alpha conditions will automatically be satisfied if the initial flow conditions and their spatial derivatives are correct at the surface.

\section{A. Time Advancing Boundary}

If the spatial derivatives are correctly specified to maintain the desired time accuracy, then after time advancing the flow variables we find that the boundary condition, Eq. (5), with $\alpha=0$ will still be satisfied as shown below (via its Taylor series expansion in time):

$$
\begin{aligned}
& n_{x} \rho(x, y, t+\Delta t) u(x, y, t+\Delta t)+ \\
& n_{y} \rho(x, y, t+\Delta t) v(x, y, t+\Delta t)= \\
& =n_{x} \rho(x, y, t) u(x, y, t)+n_{y} \rho(x, y, t) v(x, y, t)+ \\
& \quad \sum_{k=1}^{O} \frac{1}{k !}\left(n_{x} \frac{\partial^{k}(\rho u)}{\partial t^{k}}+n_{y} \frac{\partial^{k}(\rho v)}{\partial t^{k}}\right)(\Delta t)^{k} \\
& =\rho \underbrace{\left(n_{x} u(x, y, t)+n_{y} v(x, y, t)\right)}_{=0}+ \\
& \quad \sum_{k=1}^{O} \frac{1}{k !}(\sum_{i=0}^{k}\left(\begin{array}{c}
k \\
i
\end{array}\right) \frac{\partial^{k-i} \rho}{\partial t^{k-i}} \underbrace{\left[n_{x} \frac{\partial^{i} u}{\partial t^{i}}+n_{y} \frac{\partial^{i} v}{\partial t^{i}}\right]}_{=0})(\Delta t)^{k} \\
& =0
\end{aligned}
$$

At the new time step, the boundary condition, Eq. (5), for $\alpha>0$ will in general not be true. For example, we know that after time advancing the primitive variables that for $\alpha=1$ :

$$
\begin{aligned}
& n_{x}(\rho(x, y, t+\Delta t) u(x, y, t+\Delta t))_{t}+ \\
& n_{y}(\rho(x, y, t+\Delta t) v(x, y, t+\Delta t))_{t}= \\
& \quad=\quad-n_{x}\left(\rho_{x} u^{2}+2 \rho u u_{x}+p_{x}+\rho_{y} u v+\rho u_{y} v+\rho u v_{y}\right) \\
& \quad-n_{y}\left(\rho_{y} v^{2}+2 \rho v v_{y}+p_{y}+\rho_{x} u v+\rho u_{x} v+\rho u v_{x}\right) \\
& \quad \neq 0
\end{aligned}
$$

Clearly it is necessary to correct the spatial derivatives at each time step to maintain time accuracy at the boundary. In this work however, only the spatial derivatives of the pressure are corrected because this approach was successful for the $1^{s t}$ order corrections used by $\operatorname{Tam}^{7,14}$ and Hixon. ${ }^{9}$ This approach reduces the seemingly intractible complexity of very high order nonlinear boundary conditions to a few lines of code. The downside to this approach is entropy and vorticity waves may be excited as no mechanism for properly correcting the density and velocity derivatives is provided here. For example, the one-dimensional characteristic form (entropy and two acoustic waves) of the Euler equations is:

$$
\begin{aligned}
& \frac{\partial v_{i}}{\partial t}+\lambda_{i} \frac{\partial v_{i}}{\partial x}=0 \text { for } i=1,2,3 \\
& d\left(v_{1}\right)=d(\rho)-\frac{d(p)}{c^{2}}, \\
& d\left(v_{2}\right)=d(u)+\frac{d(p)}{\rho c}, \\
& d\left(v_{3}\right)=d(u)-\frac{d(p)}{\rho c} \\
& \lambda_{1}=u, \quad \lambda_{2}=u+c, \quad \lambda_{3}=u-c \\
& v_{1}=s=\text { const. for } d x=u d t \\
& v_{2}=u+\frac{2 c}{\gamma-1}=\text { const. for } d x=(u+a) d t \\
& v_{3}=u-\frac{2 c}{\gamma-1}=\text { const. for } d x=(u-a) d t
\end{aligned}
$$

where $\mathrm{c}$ is the speed of sound, $\mathrm{s}$ is the entropy, $d\left(v_{i}\right)$ is the $i^{\text {th }}$ characteristic variable differential which is integrable for isentropic flows, and $\lambda_{i}$ is the speed of the characteristic wave.

The time derivative of the Euler equations provides a new set of characteristics with the same velocities 
but include higher order space derivatives:

$$
\begin{gathered}
\frac{\partial}{\partial t}\left(\frac{\partial v_{i}}{\partial t}\right)+\left(\lambda_{i}\right)_{t} \frac{\partial v_{i}}{\partial x}+\lambda_{i} \frac{\partial}{\partial x}\left(\frac{\partial v_{i}}{\partial t}\right)=0, i=1,2,3 \\
\frac{\partial}{\partial t}\left(-\lambda_{i} \frac{\partial v_{i}}{\partial x}\right)+\lambda_{i} \frac{\partial}{\partial x}\left(-\lambda_{i} \frac{\partial v_{i}}{\partial t}\right)=\left(\lambda_{i}\right)_{t} \frac{\partial v_{i}}{\partial x} \\
d\left(v_{1}\right)=d\left(-u\left(\rho_{x}+\frac{p_{x}}{c^{2}}\right)\right) \\
d\left(v_{2}\right)=d\left(-(u+c)\left(u_{x}+\frac{p_{x}}{\rho c}\right)\right) \\
d\left(v_{3}\right)=d\left(-(u-c)\left(u_{x}-\frac{p_{x}}{\rho c}\right)\right) \\
\lambda_{1}=u, \quad \lambda_{2}=u+c, \quad \lambda_{3}=u-c
\end{gathered}
$$

where $c$ is the speed of sound. In this analysis, the lower order terms are source terms and are assumed known (using the procedure provided later).

Differentiating once more in time provides a new set of characteristics with the same velocity as before:

$$
\left.\begin{array}{c}
d\left(v_{1}\right)=d\left(u\left(u_{x}\left(\rho_{x}-\frac{p_{x}}{c^{2}}\right)-u\left(\rho_{x x}-\frac{p_{x x}}{c^{2}}\right)\right)\right) \\
d\left(v_{2}\right)=d\left((u+c)\left(u_{x}\left(u_{x}+\frac{p_{x}}{\rho c}\right)+(u+c)\left(u_{x x}+\frac{p_{x x}}{\rho c}\right)\right)\right) \\
d\left(v_{3}\right)=d\left((u-c)\left(u_{x}\left(u_{x}-\frac{p_{x}}{\rho c}\right)+(u-c)\left(u_{x x}-\frac{p_{x x}}{\rho c}\right)\right)\right)
\end{array}\right\}
$$

In Eq. (18) the entropy characteristic, $d\left(v_{1}\right)$ does not depend on velocity, but the new differentiated characteristic does depend upon velocity in Eq. (19). And, in Eq. (20) the new entropy characteristic depends on all flow variable first derivatives. By only correcting the pressure derivatives, the Euler system is underspecified because the velocity and density derivatives are incorrect at the surface. Ideally, all the flow variable derivatives would be corrected so that $d\left(v_{1}\right)$ and the outgoing acoustic characteristic are not changed. This issue is beyond the scope of this paper since it is currently standard practice to only correct the pressure terms and since physically correct solutions have been achieved with it (probably because the required corrections in the other terms were small compared to the pressure). This will become more important as higher order time accuracy is required and the procedure shown next could be extended for such cases.

\section{Surface Pressure Correction Algorithm}

The basic idea ${ }^{7}$ is to modify the normal pressure derivatives on a boundary to satisfy the governing equations and boundary conditions. One-sided derivatives at a surface will generally be incorrect since they do not account for the walls presence, thus they need to be corrected. And, contrary to the assertion of only one physical boundary condition being available for high order finite-difference schemes, we make use of the infinite additional conditions ${ }^{10}$ in Eq. (5).

For a wall with normal vector, $\vec{\eta}=\left(\eta_{x}, \eta_{y}\right)$, tangential vector, $\vec{\tau}=\left(\tau_{x}, \tau_{y}\right)$, and a numerical method with order $O$ accuracy, the pressure derivatives:

$$
\frac{\partial^{a+b} p}{\partial^{a} x \partial^{b} y} \forall(a, b):(1 \leq a+b \leq O)
$$

will be modified such that the boundary condition, Eq. (5), is satisfied while simultaneously insuring the tangential derivatives of the pressure:

$$
\frac{\partial^{\alpha} p}{\partial^{\alpha} \tau}, \frac{\partial^{\alpha} p}{\partial^{\alpha-1} \tau \partial \eta}, \ldots, \frac{\partial^{\alpha} p}{\partial \tau \partial^{\alpha-1} \eta} \forall(\alpha):(\alpha \leq O)
$$

do not change on an inviscid wall.

We can represent the new pressure, ()$_{\mathbf{N}}$, as the sum of the old, ()o, and correction, () $\mathbf{c}$, pressures:

$$
\begin{aligned}
& \left(\frac{\partial^{a+b} p}{\partial^{a} x \partial^{b} y}\right)_{\mathbf{N}}=\left(\frac{\partial^{a+b} p}{\partial^{a} x \partial^{b} y}\right)_{\mathbf{O}}+\left(\frac{\partial^{a+b} p}{\partial^{a} x \partial^{b} y}\right)_{\mathbf{C}} \\
& =\stackrel{\mathrm{o}}{C}_{a, b, 0}^{p}+\stackrel{\mathrm{C}}{\mathrm{C}}_{a, b, 0}^{\dot{p}}
\end{aligned}
$$

where

$$
\begin{aligned}
& {\stackrel{\mathrm{C}}{C_{\alpha-i, i, 0}^{p}}}^{p}= \\
& \frac{\left(-n_{x}{\stackrel{\mathrm{U}}{C_{0,0, \alpha}^{u}}}^{u}-n_{y}{\stackrel{\mathrm{U}}{C_{0,0, \alpha}^{v}}}^{\underline{\alpha}}+C_{0,0, \alpha}^{Q_{5}}\right)(-1)^{i} M_{i}}{\sum_{j=0}^{\alpha} A_{j}(-1)^{j} M_{j}} \\
& M_{i}=\sum_{b=0}^{\frac{\alpha(\alpha-1)}{2}}(-1)^{\alpha+i}\left(\frac{\frac{\alpha(\alpha-1)}{2}}{b}\right) n_{x}^{\alpha^{2}-2 b-i} n_{y}^{2 b+i} \\
& -n_{x}\left(T_{\alpha, 1, j, u, v} C_{0,0,0}^{u}+T_{\alpha, 1, j-1, u, v} C_{0,0,0}^{v}+\left(\frac{T_{\alpha, 0, j, u, v}}{C_{0,0,0}^{\rho}}\right)\right) \\
& -n_{y}\left(T_{\alpha, 1, j-1, u, v} C_{0,0,0}^{u}+T_{\alpha, 1, j-2, u, v} C_{0,0,0}^{v}+\left(\frac{T_{\alpha, 0, \alpha-j, v, u}}{C_{0,0,0}^{p}}\right)\right)
\end{aligned}
$$

$$
\begin{aligned}
& T_{\alpha, \beta, \zeta, f, g}= \\
& (-1)^{\alpha-1} \sum_{m=0}^{\frac{\zeta}{2}+1} \sum_{i=0}^{\frac{\alpha}{2}}\left[H_{m, i, \alpha, \beta, \zeta} \frac{\left(\gamma C_{0,0,0}^{p}\right)^{(i+m)}}{\left(C_{0,0,0}^{\rho}\right)^{(i+m+\beta)}}\right. \\
& \left.\quad\left(C_{0,0,0}^{f}\right)^{(\alpha-\beta-2 i-\zeta-1)}\left(C_{0,0,0}^{g}\right)^{(\zeta-2 m)}\right] \\
& \frac{(\alpha-1) !(m+i) !}{(\alpha-\beta-2 i-\zeta-1) !(\zeta-2 m) !(2(i+m)+\beta) ! i ! m !}
\end{aligned}
$$

The updated term, $\stackrel{\mathrm{U}}{C}_{0,0, \alpha}^{f}$, in Eq. (24), refers to the value of $C_{0,0, \alpha}^{f}$ after the lower order pressure derivatives have been corrected. For example, we first correct the $1^{\text {st }}(\alpha=1)$ order pressure derivatives, $\left(p_{x}, p_{y}\right)$, using Eq. (24). Next, the higher order time derivatives, $\stackrel{\mathrm{U}}{C}_{0,0,2}^{u}$ and $\stackrel{\mathrm{U}}{\mathrm{C}}_{0,0,2}^{v}$ are calculated, but using the new values of $p_{x}$ and $p_{y}$. These "updated" time derivatives are then used to calculate new $2^{\text {nd }}(\alpha=2)$ order pressure derivatives, $p_{x x}, p_{x y}, p_{y y}$, with Eq. (24).

This process is repeated until the desired accuracy is achieved. A complete derivation of Eq. (24) may be found in the appendix. 


\section{Results}

The adequacy of correcting only the pressure derivatives to achieve high time accuracy was tested by scattering the initial Gaussian pulse:

$$
p(x, y, 0)=0.001 \exp \left(-\frac{\ln 2\left[(x-1)^{2}+y^{2}\right]}{.04}\right)
$$

with four grid points per unit interval from a flat plate at progressively higher order accuracy. The initial uniform flow conditions were $\rho=1.4, p=1.0, u=0$, and $v=0$

In Fig. 2, pressure contours of the reflected pulse are shown for the $3^{r d}$ order conditions. The solution is stable, but the reflected pressure is slightly amplified. The pressure is over-corrected (amplified) because the effects of the other uncorrected variables must be accounted for to maintain the no-flow boundary conditions, Eq. (5). As the accuracy increases, the number and effect of uncorrected variables increases resulting in an over-correction of the pressure that leads to numerical instability, unless some damping mechanism is added.

In Fig. 3, the reflected pulse using the $3^{\text {rd }}$ order conditions are compared with the current practice of correcting only the first derivatives, $p_{x}$ and $p_{y}$ using $3^{r d}$ and $1^{\text {st }}$ order design time accuracy at the surface (though both achieve only $1^{\text {st }}$ order time accuracy as mentioned). The $3^{\text {rd }}$ order time accuracy cases in Figs. $3 \mathrm{a}$ and $3 \mathrm{~b}$, showed little difference here suggesting the higher order corrections are small, but the $1^{\text {st }}$ order time advance in Fig. 3c generated spurious waves and was unstable.

The $5^{\text {th }}$ order example in Fig. 4 shows more clearly the importance of correcting the higher derivatives. Despite only correcting the pressure, the reflected pulse is qualitatively superior to correcting only the first order pressure derivatives. The simulation becomes unstable however on the surface after the pulse has reflected from it at time $t=0.8$. Higher order conditions result in larger over-corrections of the pressure, as expected. Note that the MESA scheme explicitly uses the $2^{n d}$ order spatial derivatives and the effects of high order boundary corrections are directly observable. Other methods may perform differently for the cases shown in Fig. $4 \mathrm{~b}$ and $4 \mathrm{c}$, , though their results remain nonphysical.

As a test of the procedures on curved surfaces with a Cartesian grid, the same Gaussian pulse was scattered from a sphere of radius, $r=.25$, with a grid density of 128 points per unit interval. In Fig. 5, a $3^{\text {rd }}$ order solution is shown. The curvature results in greater changes in the velocity and the pressure corrections must compensate for that, resulting in a dissipated, but clearly reflected pulse and was numerically stable. The higher order conditons $(>4)$ were unstable without adding artificial dissipation for the circle case.
As a more realistic example, observe the entropy production shown for the $2 \mathrm{D}$ stator blade passage in Fig. 6 in which the height of the grid indicates magnitude of the entropy. Only the $1^{\text {st }}$ order pressure derivatives are corrected and for this inviscid problem the solution should be flat, indicating no change in entropy. Here, the curvlinear form of the nonlinear Euler equations are solved using a $6^{\text {th }}$ order compact scheme $^{18}$ for spatial derivatives and a $4^{\text {th }}$ order 5-6 Low Dispersion and Dissipation Runge-Kutta scheme for time marching. ${ }^{19}$ Notice the entropy production at the suction surface and convected downstream in the wake.

Overall, the advantages of correcting the high order spatial derivatives is clear, and as shown in Fig. 1, the efficiency of high accuracy is evident for demanding long-term low error tolerance simulations. But the current practice of correcting only the pressure is not physically correct despite the flow variables satisfying the governing equations and boundary conditions at the surface. The practice is still nonphysical (e.g., produces entropy and vorticity) because the characteristics are under-specified and therefore the other flow variables must be corrected as well.

\section{Conclusion}

Most surface boundary methods in use today achieve only $1^{\text {st }}$ order accuracy in time since only the no flow condition is imposed. Higher time accuracy requires enforcing the highier order time derivatives of the no flow condition. In the special case of stationary surfaces, the even ordered time derivatives of the no flow condition will be automatically satisfied if they were correctly specified at the start of the simulation.

The primary advantage of high time accuracy is better resolution and larger time steps at surfaces. Achieving high time accuracy requires correcting cross-derivatives of the flow at a surface. In principle, any numerical method could be used with the boundary procedure presented here. But a Hermitian MESA scheme was used because of its extraordinary resolution and potential for efficiency at a surface with high time accuracy. The exact performance advantages of high time accuracy will depend upon the physical accuracy required.

The design time accuracy was increased here by correcting only the pressure as is the currently accepted $1^{\text {st }}$ order practice. Despite the apparently high complexity in deriving very high order pressure corrections, a simple process for doing this was found that requires only a few lines of code to implement. However, a characteristic analysis and numerical testing has revealed that it is important to correct the velocity and density spatial derivatives as well. Therefore, an important next step is to correct all flow derivatives to avoid underspecifying the hyperbolic Euler system and to extend this analysis to curvilinear coordinates 


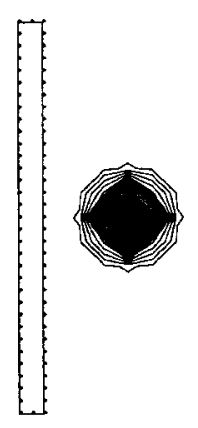
a) $t=0$

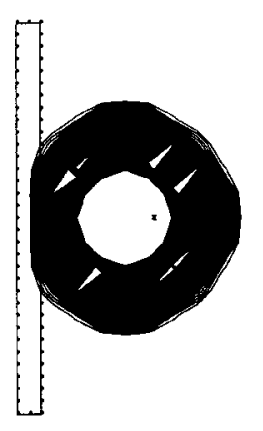

b) $t=.3$

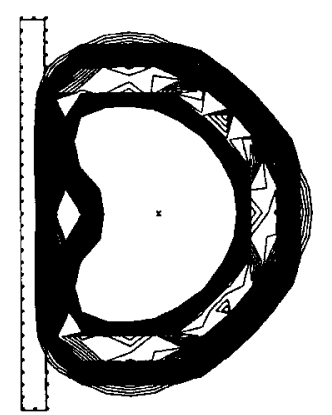

c) $\mathrm{t}=.6$

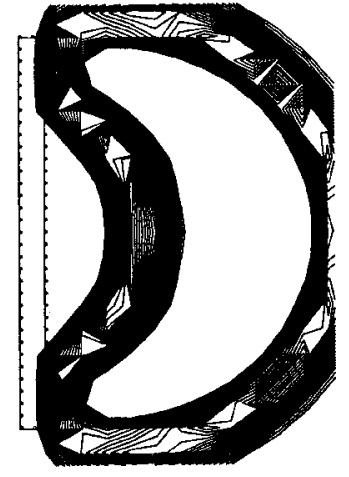

d) $\mathrm{t}=\mathbf{1}$

Fig. 2 Third Order Scattered Pulse From Plate

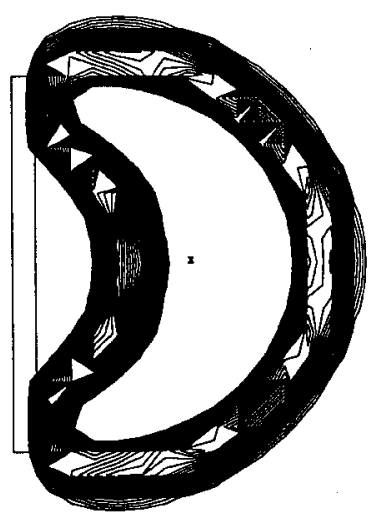

a) Fully $3^{\text {rd }}$ Order in Time

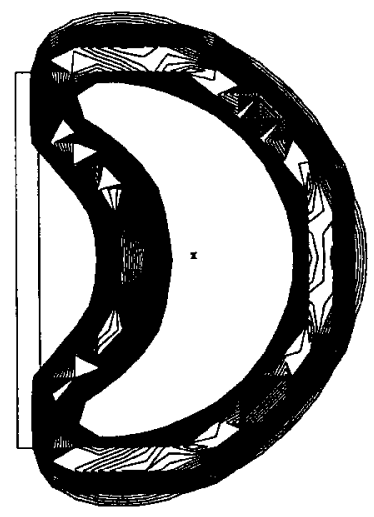

b) Partially $3^{\text {rd }}$ Order in Time

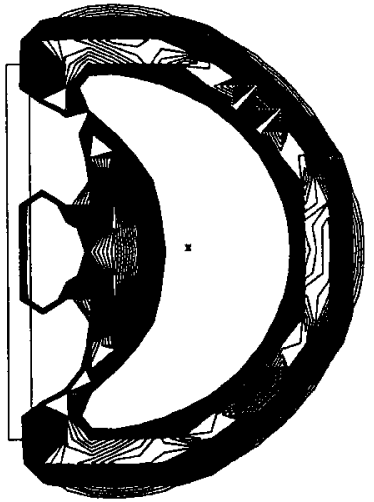

c) Partially $1^{\text {st }}$ Order in Time

Fig. 3 Fully VS. Partially Corrected $3^{\text {rd }}$ Order
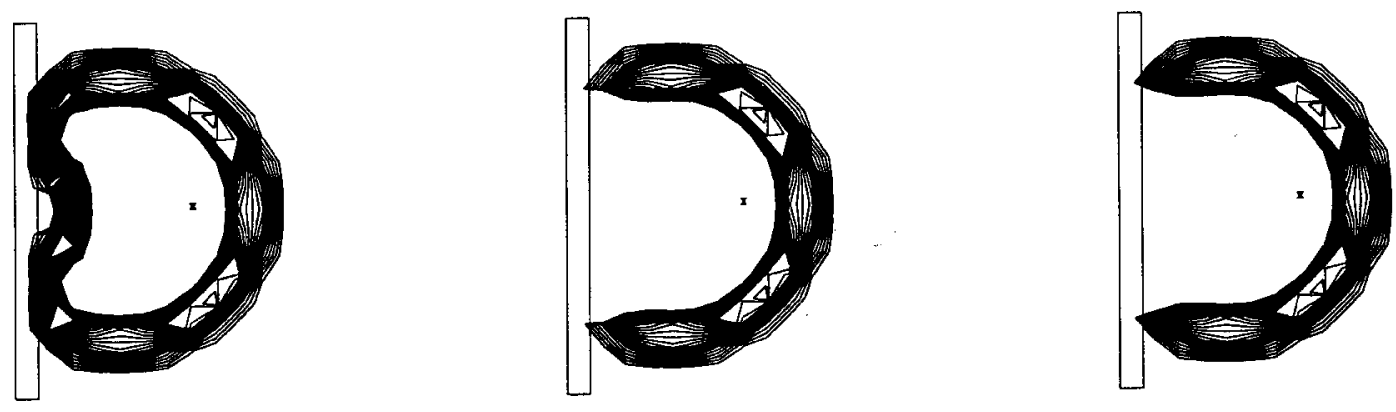
a) Fully $5^{\text {th }}$ Order in Time
b) Partially $5^{\text {th }}$ Order in Time
c) Partially $1^{\text {st }}$ Order in Time

Fig. 4 Fully VS. Partially Corrected $5^{\text {th }}$ Order 


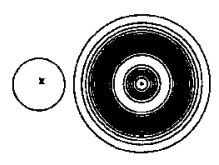

a) $\mathbf{n}=190$
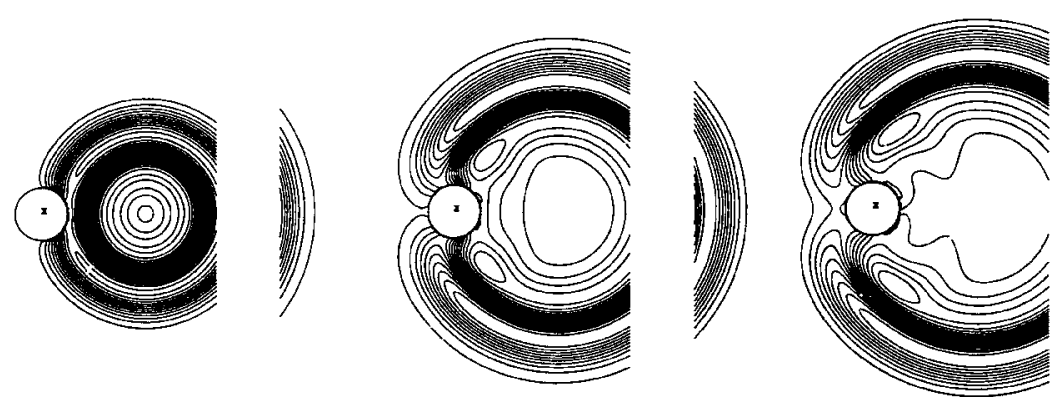

d) $n=1410$

Fig. 5 Third Order Pulse Scattered From Circle

to efficiently resolve curvature effects.

\section{Appendix: Derivation of Pressure Correction}

The pressure correction procedure shown in section IV was derived by assuming only the pressure's spatial derivatives required adjustment to account for the presence of the wall. After the corrections are applied, the flow variables and their spatial derivatives must satisfy the nonlinear Euler equations and the boundary condition at the surface. In addition, the flow's spatial derivatives must satisfy the time derivatives of these equations to achieve high accuracy in time.

Starting with the following boundary condition, from Eq. (5), for any $\alpha$ :

$$
\frac{\partial^{\alpha}(\vec{V} \cdot \hat{n})}{\partial t^{\alpha}}=n_{x} \frac{\partial^{\alpha} u}{\partial t^{\alpha}}+n_{y} \frac{\partial^{\alpha} v}{\partial t^{\alpha}}=C_{0,0, \alpha}^{Q_{5}}
$$

we want to correct the pressure derivatives so that the following is satisfied:

$n_{x}\left(\stackrel{\stackrel{\mathrm{O}}{C}}{\mathrm{u}}_{0,0, \alpha}+\stackrel{\mathrm{C}}{\mathrm{C}}_{0,0, \alpha}^{u}\right)+n_{y}\left(\stackrel{\mathrm{o}}{\mathrm{C}}_{0,0, \alpha}+\stackrel{\mathrm{C}}{\mathrm{C}_{0,0, \alpha}^{v}}\right)=C_{0,0, \alpha}^{Q_{5}}$

The terms, $\stackrel{\mathrm{C}}{\mathrm{C}}_{0,0, \alpha}$ and $\stackrel{\mathrm{C}}{\mathrm{C}}_{0,0, \alpha}$, are the corrections to the time derivatives of the velocities resulting from the corrections to the spatial derivatives of the pressure. We need to determine how changes in the pressure derivatives will effect the velocity time derivatives so that Eq. (30) is satisfied up to some specified order $\alpha$.

This relationship can be found using the governing equations, Eqs. $(1,2,3,4)$ and the following alternate form of the momentum equations: ${ }^{16}$

$$
\begin{aligned}
& \frac{\partial u}{\partial t}=-\left(u \frac{\partial u}{\partial x}+v \frac{\partial u}{\partial y}+\frac{1}{\rho} \frac{\partial p}{\partial x}\right) \\
& \frac{\partial v}{\partial t}=-\left(u \frac{\partial v}{\partial x}+v \frac{\partial v}{\partial y}+\frac{1}{\rho} \frac{\partial p}{\partial y}\right)
\end{aligned}
$$

From this alternate momentum equation form we find the following relationship:

$$
\begin{aligned}
& \stackrel{\mathbf{C}}{C}_{0,0, \alpha}^{u}= \\
& -\left(C_{0,0,0}^{u} \stackrel{\mathbf{C}_{1,0, \alpha-1}^{u}}{\mathbf{C}_{u, 0,0}} C_{0,1, \alpha-1}^{v}+\frac{1}{C_{0,0,0}^{\rho}} \stackrel{\mathrm{C}}{C}_{1,0, \alpha-1}^{u}\right) \\
& \stackrel{\mathrm{C}}{\mathrm{C}}_{0,0, \alpha}= \\
& -\left(C_{0,0,0}^{u} \stackrel{\mathbf{C}_{1,0, \alpha-1}^{v}}{{ }^{v}}+C_{0,0,0}^{v} \stackrel{\mathbf{C}_{0,1, \alpha-1}^{v}}{C_{0,0,0}}+\frac{1}{C_{0,1, \alpha-1}^{\rho}}\right)
\end{aligned}
$$

which shows how changes in pressure and velocity mixed space and time derivatives will affect the boundary condition equation.

The critical and most difficult next step in this derivation is expressing the right hand side of Eq. (31) in terms of the corrected pressure's spatial derivatives. This was done by recursively differentiating all six governing equation forms with computer algebra until a pattern could be discerned. The following pattern was observed and inductively verified:

$$
\begin{aligned}
& {\stackrel{\mathrm{C}}{C_{1,0, \alpha-1}^{u}}}^{u}=\sum_{j=0}^{\alpha}{\stackrel{\mathrm{C}}{C_{\alpha-j, j, 0}^{p}}}_{T_{\alpha, 1, j, u, v}} \\
& {\stackrel{\mathrm{C}}{C_{0,1, \alpha-1}^{u}}}^{u}=\sum_{j=0}^{\alpha}{\stackrel{\mathrm{C}}{C_{\alpha-j, j, 0}^{p}}}_{T_{\alpha, 1, j-1, u, v}} \\
& \stackrel{\mathrm{C}}{\mathrm{C}}_{1,0, \alpha-1}^{v}=\sum_{j=0}^{\alpha}{\stackrel{\mathrm{C}}{C_{\alpha-j, j, 0}^{p}}}_{T_{\alpha, 1, j-1, u, v}} \\
& {\stackrel{\mathrm{C}}{C_{0,1, \alpha-1}^{v}}}^{v}=\sum_{j=0}^{\alpha}{\stackrel{\mathrm{C}}{C_{\alpha-j, j, 0}^{p}}}_{T_{\alpha, 1, j-2, u, v}} \\
& \stackrel{\mathrm{C}}{\mathrm{C}}_{1,0, \alpha-1}^{p}=\sum_{j=0}^{\alpha} \stackrel{\mathrm{C}}{\mathrm{C}}_{\alpha-j, j, 0}^{p} T_{\alpha, 0, j, u, v} \\
& {\stackrel{\mathrm{C}}{C_{0,1, \alpha-1}}}_{p}=\sum_{j=0}^{\alpha}{\stackrel{\mathrm{C}}{C_{\alpha-j, j, 0}^{p}}}^{p} T_{\alpha, 0, \alpha-j, v, u}
\end{aligned}
$$

where $T_{\alpha, \beta, \zeta, f, g}$ is defined in Eq. (27).

For each value of $\alpha$, we can now correct the following pressure derivatives:

$$
\stackrel{\mathrm{C}}{\mathrm{C}}_{\alpha-j, j, 0} \forall j=0,1, \ldots, \alpha
$$




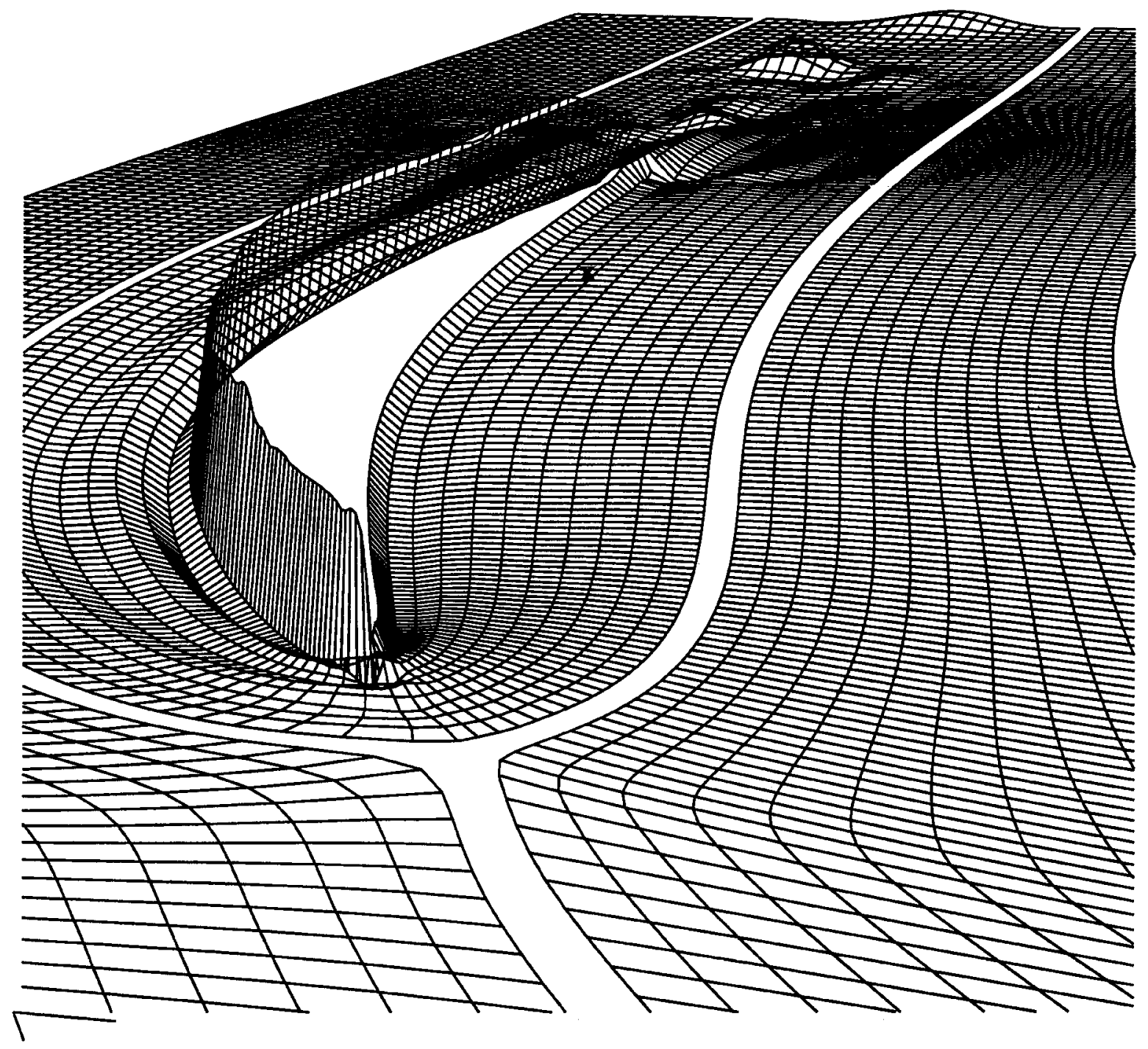

Fig. 6 Entropy Produced on Surface of Stator Blade On Curvilinear Grid 
by subsituting Eq. (33) in to Eq. (32) and solving boundary condition Eq. (30):

$$
\sum_{j=0}^{\alpha} \stackrel{\mathrm{C}}{C}_{\alpha-j, j, 0}^{p} A_{j}=C_{0,0, \alpha}^{Q_{5}}-n_{x} \stackrel{\mathrm{U}}{\mathrm{C}}_{0,0, \alpha}^{u}-n_{y} \stackrel{\mathrm{U}}{\mathrm{C}}_{0,0, \alpha}^{v}
$$

with $A_{j}$ defined in Eq. (26).

In addition, since we do not want corrections of the pressure derivative to effect the tangential derivatives, we impose, for each $\alpha$ the following $\alpha$ conditions :

$$
\left(\frac{\partial^{\alpha} p}{\partial \tau^{\alpha-j} \partial \eta^{j}}\right)_{\text {correction }}=0 \forall j: j=0,1, \ldots, \alpha-1
$$

which can be written as a condition of only the Cartesian $\mathrm{x}$ and $\mathrm{y}$ pressure derivatives as:

$$
\begin{aligned}
& \left(\frac{\partial^{\alpha} p}{\partial \tau^{\alpha-j} \partial \eta^{j}}\right)_{\text {correction }}= \\
& =\left(\tau_{x} \frac{\partial}{\partial x}+\tau_{y} \frac{\partial}{\partial y}\right)^{\alpha-j}\left(\eta_{x} \frac{\partial}{\partial x}+\eta_{y} \frac{\partial}{\partial y}\right)^{j} p \\
& =\sum_{a=0}^{\alpha}{\stackrel{\mathbf{C}}{C_{\alpha-a, a, 0}}}^{p} B_{a, j} \\
& =0
\end{aligned}
$$

with

$$
\begin{aligned}
& B_{a, j}=\sum_{b=0}^{j}\left(\begin{array}{c}
\alpha-j \\
a-b
\end{array}\right)\left(\begin{array}{l}
j \\
b
\end{array}\right) \\
& (-1)^{(b-a)}\left(n_{x}\right)^{(j-2 b+a)}\left(n_{y}\right)^{(\alpha-j+2 b-a)}
\end{aligned}
$$

since $\tau_{x}=\eta_{y}$ and $\tau_{y}=-\eta_{x}$.

For each value of $\alpha$, we can write the $\alpha+1$ conditions in Eqs. $(35,37)$ in the following matrix form:

$$
\begin{aligned}
& \underbrace{\left[\begin{array}{cccc}
A_{0} & A_{1} & \ldots & A_{\alpha} \\
B_{0,0} & B_{1,0} & \ldots & B_{\alpha, 0} \\
B_{0,1} & B_{1,1} & \ldots & B_{\alpha, 1} \\
B_{0,2} & B_{1,2} & \ldots & B_{\alpha, 2} \\
\vdots & \vdots & \ddots & \vdots \\
B_{0, \alpha-1} & B_{1, \alpha-1} & \ldots & B_{\alpha, \alpha-1}
\end{array}\right]}_{E} \underbrace{\left[\begin{array}{l}
\stackrel{C}{C}_{\alpha, 0,0}^{p} \\
\stackrel{C}{C}_{\alpha-1,1,0}^{p} \\
\stackrel{C}{C}_{\alpha-2,2,0}^{p} \\
\vdots \\
\mathbf{C}_{0, \alpha, 0}^{p}
\end{array}\right]}_{C} \\
& =\underbrace{\left[\begin{array}{c}
C_{0,0, \alpha}^{Q_{5}}-n_{x} C_{0,0, \alpha}^{u}-n_{y} C_{0,0, \alpha}^{v} \\
0 \\
0 \\
0 \\
\vdots \\
0
\end{array}\right]}_{D}
\end{aligned}
$$

Since the right hand side of this matrix system is zero, except the first term, we can efficiently solve this system with Cramer's rule for the $i^{t h}$ element of vector $C$ :

$$
C_{i}=\frac{\operatorname{det}(F)}{\operatorname{det}(E)}
$$

where matrix $F$ equals matrix $E$ with column number i replace by vector $D$.

The determinant of a matrix may be solved using cofactor expansion along a column. ${ }^{17}$ So we can take advantage of all the zeros in $F$ by expanding along column i to efficiently evaluate the determinant of the matrix:

$$
\operatorname{det}(F)=\overbrace{\left(C_{0,0, \alpha}^{Q_{5}}-n_{x} \stackrel{U}{C}_{0,0, \alpha}^{u}-n_{y}{\stackrel{U}{C_{0,0, \alpha}^{v}}}^{D_{1}}\right.}^{D_{1}} \overbrace{(-1)^{i} M_{i}}^{\text {cofactor }}
$$

where $M_{i}$ is the minor of the element in the first row and $i^{t h}$ column of matrix $F$.

Similarly we can evaluate the determinant of matrix $E$ by cofactor expansion along the top row:

$$
\begin{aligned}
& \operatorname{det}(E)=\sum_{j=0}^{\alpha} A_{j}(-1)^{j} M_{j} \\
& =(-1)^{\alpha}\left(n_{x}^{2}+n_{y}^{2}\right)^{\frac{\alpha(\alpha-1)}{2}}\left(\sum_{i=0}^{\alpha} n_{x}^{\alpha-i} n_{y}^{i} A_{i}\right)
\end{aligned}
$$

where $A_{j}$ is defined in Eq. (26). The expression for $M_{j}$, from Eq. (25), was derived by observation with the help of computer algebra and verified inductively.

With $M_{j}$ known, we can quickly apply Cramer's rule to solve Eq. (39) for the unknown pressure correction vector $C$ in Eq. (38). This simple procedure is repeated for $\alpha=1,2, \ldots, O$ until all higher order pressure spatial derivatives are corrected.

\section{References}

${ }^{1}$ Tam, C. K. W., "Computational Aeroacoustics: Issues and Methods", AIAA Journal, Vol. 33, No. 10, 1995, pp. 1788-1796.

${ }^{2}$ Lele, S. K., "Computational Aeroacoustics: A Review", AIAA Paper 97-0018, Jan. 1997

${ }^{3}$ Mankbadi R. R. "Review of computational aeroacoustics in propulsion systems", Journal of Propulsion and Power, Vol. 15, No. 4, 1999, pp. 504-512

${ }^{4}$ Dyson, R.W., Goodrich, J.W. "Automated Approach to Very High-Order Aeroacoustic Computations", $A I A A$ Journal, Vol. 39, No., 3, pp. 396-406

${ }^{5}$ Dyson, R.W. "Technique for Very High Order Nonlinear Simulation and Validation", Journal of Computational Acoustics, Vol. 10, No. 2, June 2002.

${ }^{6}$ Dadone, A., Grossman, B. "Surface Boundary Conditions for the Numerical Solution of the Euler Equations", AIAA J., Vol. 32, No. 2, February, 1994.

${ }^{7}$ Tam, C.K.W., and Dong, Z. "Wall Boundary Conditions for High-Order Finite Difference Schemes in Computational Aeroacoustics", AIAA Paper 94-0457, Reno, NV, Jan. 1994.

${ }^{8}$ Tam, C.K.W., "Advances in Numerical Boundary Conditions for Computational Aeroacoustics", J. of Computational Acoustics, Vol. 6, No. 4, 1998, pp. 377-402

${ }^{9}$ Hixon, R. "Curvilinear Wall Boundary Conditions for Computational Aeroacoustics", AIAA Paper 99-2395, 35th AIAA/ASME/SAE/ASEE Joint Propulsion Conference and Exhibit, Los Angeles, CA, June 1999.

${ }^{10}$ Goodrich, J.W. "High Order Implementations of Accurate Boundary Conditions", AIAA 99-1942, 1999. 
${ }^{11}$ Hixon, R. "Nonlinear Comparison of High-order and Optimized Finite-difference Schemes", Int. J. Comp. Fluid Dynamics, Vol. 13, 2000, pp. 259-277

${ }^{12}$ Gustaffson, B. "The Convergence Rate for Difference Approximations to Mixed Initial Boundary Value Problems", Mathematics of Computations, Vol. 29, 1975, pp. 396-406

${ }^{13}$ Casper, J., and Carpenter, M.H. "Computational Considerations for the Simulation of Shock-Induced Sound", Siam Journal on Scientific Computing, Vol. 19 No. 3, May 1998, pp. 813-828

${ }^{14}$ Kurbatskii, K.A., Tam, C.K.W. "Cartesian Boundary Treatment of Curved Walls for High-Order Computational Aeroacoustics Schemes", AIAA J., Vol. 35, No. 1, January 1997, pp. $133-140$

${ }^{15}$ Goodrich, J.W. "A Comparison of Numerical Methods for Computational Aeroacoustics", 5th AIAA/CEAS Aeroacoustics Conference, AIAA 99-1943, May 1999.

${ }^{16}$ Anderson, J.D., Fundamentals of Aerodynamics, McGraw-Hill Book Company, 1984, pp.472.

${ }^{17}$ Anton, H. Elementary Linear Algebra, 5th ed., John Wiley \& Sons, 1987 , p. 85

${ }^{18}$ Hixon, R., "A New Class of Compact Schemes", AIAA Paper 98-0367, Reno, NV, January 1998

${ }^{19}$ Stanescu, D., and Habashi, W.G., "2N-Storage Low Dissipation and Dispersion Runge-Kutta. Schemes for Computational Aeroacoustics", Journal of Computational Physics, Vol. 143, pp. 674-681, 1998 


\section{\begin{tabular}{l|l|l|} 
1. AGENCY USE ONLY (Leave blank) & 2. REPORT DATE & 3. REPORT TYPE AND DATES COVERED
\end{tabular}}

4. TITLE AND SUBTITLE

July 2002

Technical Memorandum

5. FUNDING NUMBERS

Towards Arbitrary Accuracy Inviscid Surface Boundary Conditions

6. AUTHOR(S)

Rodger W. Dyson and Ray Hixon

\section{PERFORMING ORGANIZATION NAME(S) AND ADDRESS(ES)}

National Aeronautics and Space Administration

John H. Glenn Research Center at Lewis Field

Cleveland, Ohio 44135-3191

WU-780-30-11-00

9. SPONSORING/MONITORING AGENCY NAME(S) AND ADDRESS(ES)

National Aeronautics and Space Administration

Washington, DC 20546-0001

8. PERFORMING ORGANIZATION

REPORT NUMBER

E-13368

10. SPONSORING/MONITORING AGENCY REPORT NUMBER

NASA TM-2002-211583

ICOMP-2002-04

AIAA-2002-2438

11. SUPPLEMENTARY NOTES

Prepared for the Eighth Aeroacoustics Conference cosponsored by the American Institute of Aeronautics and Astronautics and the Confederation of European Aerospace Societies, Breckenridge, Colorado, June 17-19, 2002. Rodger W. Dyson, NASA Glenn Research Center; and Ray Hixon, Senior Research Associate, Institute for Computational Mechanics in Propulsion, Cleveland, Ohio and University of Toledo, Toledo, Ohio 43606. Responsible person, Rodger W. Dyson, organization code 5940, 216-433-9083.

\begin{tabular}{l|l}
\hline 12a. DISTRIBUTION/AVAILABILITY STATEMENT & 12b. DISTRIBUTION CODE
\end{tabular}

Unclassified - Unlimited

Subject Category: 71

Distribution: Nonstandard

Available electronically at htp//gltrs.grenasa.gov/GLTRS

This publication is available from the NASA Center for AeroSpace Information, 301-621-0390.

13. ABSTRACT (Maximum 200 words)

Inviscid nonlinear surface boundary conditions are currently limited to third order accuracy in time for non-moving surfaces and actually reduce to first order in time when the surfaces move. For steady-state calculations it may be possible to achieve higher accuracy in space, but high accuracy in time is required for efficient simulation of multiscale unsteady phenomena. A surprisingly simple technique is shown here that can be used to correct the normal pressure derivatives of the flow at a surface on a Cartesian grid so that arbitrarily high order time accuracy is achieved in idealized cases. This work demonstrates that nonlinear high order time accuracy at a solid surface is possible and desirable, but it also shows that the current practice of only correcting the pressure is inadequate.

\begin{tabular}{|c|c|c|c|}
\hline \multirow{2}{*}{\multicolumn{3}{|c|}{$\begin{array}{l}\text { 14. SUBJECT TERMS } \\
\text { Nonlinear Euler; MESA; High resolution; Efficiency; Computational aeroacoustics; } \\
\text { Characteristics }\end{array}$}} & 15. NUMBER OF PAGES \\
\hline & & & 16. PRICE CODE \\
\hline $\begin{array}{l}\text { 17. SECURITY CLASSIFICATION } \\
\text { OF REPORT }\end{array}$ & $\begin{array}{l}\text { 18. SECURITY CLASSIFICATION } \\
\text { OF THIS PAGE }\end{array}$ & $\begin{array}{l}\text { 19. SECURITY CLASSIFICATION } \\
\text { OF ABSTRACT }\end{array}$ & 20. LIMITATION OF ABSTRACT \\
\hline Unclassified & Unclassified & Unclassified & \\
\hline
\end{tabular}

